\title{
Polyphenol Diversity and Antioxidant Activity of European Cistus creticus L. (Cistaceae) Compared to Six Further, Partly Sympatric Cistus Species
}

\author{
Brigitte Lukas *, Laura Bragagna (D), Katharina Starzyk (D), Klaudia Labedz, Klaus Stolze and Johannes Novak \\ Institute of Animal Nutrition and Functional Plant Compounds, University of Veterinary Medicine Vienna, \\ Veterinärplatz 1, 1210 Vienna, Austria; laura.bragagna@gmail.com (L.B.); a01005378@unet.univie.ac.at (K.S.); \\ klaudia.lab@gmail.com (K.L.); klaus.stolze@drei.at (K.S.); Johannes.Novak@vetmeduni.ac.at (J.N.) \\ * Correspondence: Brigitte.Lukas@vetmeduni.ac.at; Tel.: +43-1-25077-3110; Fax: +43-1-25077-3190
}

\section{check for}

updates

Citation: Lukas, B.; Bragagna, L.; Starzyk, K.; Labedz, K.; Stolze, K.; Novak, J. Polyphenol Diversity and Antioxidant Activity of European Cistus creticus L. (Cistaceae) Compared to Six Further, Partly Sympatric Cistus Species. Plants 2021, 10, 615. https://doi.org/10.3390/ plants10040615

Academic Editor: Antonella Smeriglio

Received: 3 March 2021

Accepted: 21 March 2021

Published: 24 March 2021

Publisher's Note: MDPI stays neutral with regard to jurisdictional claims in published maps and institutional affiliations.

Copyright: (c) 2021 by the authors. Licensee MDPI, Basel, Switzerland. This article is an open access article distributed under the terms and conditions of the Creative Commons Attribution (CC BY) license (https:/ / creativecommons.org/licenses/by/ $4.0 /)$.
Abstract: This investigation focused on the qualitative and quantitative composition of polyphenolic compounds of Mediterranean northern shore Cistus creticus and six further, partly sympatric Cistus species (C. albidus, C. crispus, C. ladanifer, C. monspeliensis, C. parviflorus, C. salviifolius). Aqueous extracts of 1153 individual plants from 13 countries were analyzed via high performance liquid chromatography (HPLC). The extracts of $C$. creticus were primarily composed of two ellagitannins (punicalagin and punicalagin gallate) and nine flavonol glycosides (myricetin and quercetin glycosides, with m-3-O-rhamnoside as the dominant main compound). Differences in the proportions of punicalagin derivatives and flavonol glycosides allowed the classification into two chemovariants. Plants containing punicalagin derivatives and flavonol glycosides were especially abundant in the western and central Mediterranean areas and in Cyprus. From Albania eastwards, punicalagin and punicalagin gallate were of much lesser importance and the predominant chemovariant there was a nearly pure flavonol type. With its two chemovariants, C. creticus takes a central position between the flavonol-rich, purple-flowered clade (besides C. creticus, here represented by C. albidus and C. crispus) and the more ellagitannin-rich, white- or whitish-pink-flowered clade (here represented by $C$. ladanifer, C. monspeliensis, C. parviflorus and C. salviifolius). The median antioxidative capacity of $C$. creticus plant material was, with $166 \mathrm{mg}$ Trolox equivalents/g dry wt, about half of the antioxidative capacity of $C$. ladanifer ( $301 \mathrm{mg}$ te/g dry wt), the species with the highest antioxidative potential.

Keywords: Cistus creticus; Cistaceae; rockrose; HPLC; polyphenols; flavonoids; ellagitannins; antioxidant activity; chemotaxonomy

\section{Introduction}

Cistus L. (Cistaceae, Malvales) comprises about 20 frutescent and suffrutescent shrub species distributed in the Mediterranean, on the Canary Islands and on Madeira. The genus is taxonomically complex, and hybridization and a high degree of morphological polymorphism complicate the determination of species boundaries. Various monographs have recognized between 16 and 28 species and have proposed conflicting intrageneric classifications. The latest taxonomic treatments based on molecular phylogenetics and pollen analyses recognized a well-supported, purple-flowered clade (equivalent to subgenus Cistus and including all pink-flowered Cistus species except for C. parviflorus) and a second, sometimes weakly supported white- and whitish-pink-flowered clade (comprising the two subgenera Leucocistus and Halimioides and C. parviflorus) [1,2]. Cistus creticus L. (syn. C. incanus auct., C. villosus L.) is a prominent member of the smaller, purple-flowered clade and one of the few Cistus species widely distributed in the eastern Mediterranean. The species seems to be a good taxonomic entity but appears to be highly variable with some geographical structuring. The high morphological variability is reflected in the plethora of scientific names given to the presently recognized C. creticus to distinguish the various 
kinds of variation. The specific name C. creticus seems to be widely accepted (Euro+Med PlantBase, [3]) but the putative synonyms $C$. incanus and C. villosus have been frequently applied in local floras and the recent literature (see list of references). Based on morphological and phytochemical characteristics, some authors recognized three subspecies, Cistus creticus subsp. eriocephalus (Viv.) Greuter and Burdet, C. creticus subsp. corsicus (Loisel.) Greuter and Burdet (both poor in essential oil) and C. creticus subsp. creticus, rich in essential oil (e.g., [4-6]).

Amongst other Cistus species, $C$. creticus has been known as a medicinal plant since ancient times. Ladanum, exudates from the glandular hairs of the stem and leaves, or herbal infusions from leaves and upper stems have been used to heal eczema, abscesses, furuncles and diarrhea or to treat hair loss (e.g., [7,8]). Today, herbal infusions, extracts or cremes are consumed or applied for the treatment of, e.g., influenza, respiratory disorders, borrelioses or skin irritations. The diverse positive health effects observed can be attributed to a wide spectrum of secondary compounds. The essential oil of $C$. creticus is rich in labdane-type diterpenes (e.g., $[5,6,9,10])$. Additionally, the presence of a wide range of non-volatile compounds, mainly belonging to phenolic acids, ellagitannins and flavonols, was described [11-14]. There is thus considerable interest in this species and a vast number of publications present diverse pharmacological activities, e.g., antioxidant (e.g., [12,15]), antiinflammatory [16], antiviral (e.g., [17-19]), antimicrobial (e.g., [14,20-24]), anticancer [25-27], cardiovascular protecting [28] or skin protecting (e.g., [29,30]) activities.

Pharmacological properties and, subsequently, health benefits of plant preparations, however, are strongly dependent on their secondary compound composition. Despite the increasing knowledge of promising properties of $C$. creticus plant material, the natural variability of the responsible compounds is largely unknown. Pharmacologically, methodically or more compound characterization-orientated investigations of $C$. creticus plant material (e.g., [31-33]) often rely on trade samples or plant material from just one or a few pooled individual plants. In many cases, plant material used for the studies was not chemically characterized. A comprehensive overview is also hindered by the confusing taxonomic circumstances and the use of different methodical approaches that impede a comparison and summary of results. The few previous studies on natural biodiversity of $C$. creticus presented either uncharacterized NMR data [34] or were focused on the essential oil composition (e.g., $[5,6,9,10])$. Not much is known about abundance and intraspecific variability of non-volatile secondary compounds present in C. creticus. The authors of [11] investigated polyphenolic compounds of one or a few individual plants of ten different Cistus species native to Spain (among them C. creticus) and provided a first comparative overview about intrageneric diversity and species-specific characteristics of phenolic acid derivatives, ellagitannins and flavonoids. Two more publications described the flavonol and punicalagin/punicalagin gallate diversity of Cypriot C. creticus [35] or comparatively discussed non-volatile compounds of Sardinian C. creticus belonging to different subspecies [14]. The main aim of this investigation was to expand the fragmentary knowledge by comparatively characterizing flavonol compound diversity of natural populations of $C$. creticus, $C$. albidus, C. crispus (all three from the purple-flowered clade), C. ladanifer, C. monspeliensis, C. parviflorus and C. salviifolius (four species of the white- and whitish-pink-flowered clade). Based on our specific experimental procedure, beside flavonols, two more compounds belonging to the ellagitannin compound family, punicalagin and punicalagin gallate, were prominently present in C. creticus and were included in the comparative analysis. Two additional parameters, antioxidant activity (2,2-diphenyl-1-picrylhydrazyl (DPPH)) and total phenolic content, were determined to define and comparatively describe drug activity. One thousand one hundred and fifty-three individual plants of altogether 127 populations from 13 Mediterranean countries were studied for their total phenolic content, compound composition and antioxidant activity. Additionally, 15 commercial samples were analyzed to verify the labeling and quality of Cistus products for medical applications. The results presented here contribute to the knowledge of chemical characteristics and inter- and intraspecific compound diversity of promising medicinal plants, may provide arguments 
for chemosystematic considerations as well as evidence for pharmacologists and may therewith finally help to improve drug activity and product safety.

\section{Results}

In total, one hundred and twenty-seven populations (1153 individual plants) of 8 different Cistus taxa were analyzed. Cistus creticus was paramount (72 populations, 704 individual plants), accompanied by C. albidus (13 populations, 131 individual plants), C. $x$ canescens (one population, one plant), C. crispus (two populations, 22 individual plants), C. ladanifer (one population, eight plants), C. monspeliensis (eight populations, 89 individual plants), C. parviflorus (eight populations, 48 individual plants) and C. salviifolius (22 populations, 150 individual plants).

\subsection{Extract Composition and Chemotypes}

Chromatogram evaluation was performed at $354 \mathrm{~nm}$ focusing on conspicuous and recurrent peaks present in the species of main interest, C. creticus. Altogether, 13 major peaks (Table 1; Figure S1, Supplementary Material) representing between 55 and $100 \%$ of the total HPLC peak area (75 to $100 \%$ in C. creticus, 70 to $100 \%$ in C. albidus, 91 to $97 \%$ in C. crispus, 80 to $96 \%$ in C. ladanifer, 65 to $93 \%$ in C. monspeliensis, 55 to $90 \%$ in C. parviflorus and 60 to $100 \%$ in C. salviifolius; data not shown) were analyzed. Among these 13 peaks were nine flavonols, four myricetin glycosides (m-3-O-galactoside, m-3-O-glucoside, $\mathrm{m}$ $\mathrm{O}$-xyloside or $\mathrm{m}-3-\mathrm{O}$-arabinoside, $\mathrm{m}-3-\mathrm{O}$-rhamnoside) and five quercetin glycosides ( $\mathrm{q}$ 3-O-rutinoside, q-3-O-galactoside, q-3-O-glucoside, q-O-xyloside or q-3-O-arabinoside, $\mathrm{q}-3$-O-rhamnoside). Based on compound tables of the relevant literature, we initially focused on such myricetin and quercetin glycosides and were surprised by the appearance of four additional prominent peaks that eluted much earlier and exhibited distinct UV spectra. These four peaks were subsequently identified as punicalagin derivatives, more specifically two punicalagin isomers and two punicalagin gallate isomers (Table 1).

Table 1. HPLC retention times (Rt) of the 13 evaluated peaks, mass data (base ions at negative mode $\left([\mathrm{M}-\mathrm{H}]^{-}\right)$, main fragment ions (MS/MS)), peak identification ( $\mathrm{M}=$ myricetin, $\mathrm{Q}=$ quercetin) and relevance of compounds within aqueous extracts of $C$. creticus (range of relative area percentages at $354 \mathrm{~nm}$ ). ${ }^{1}$ Highlights compounds that were identified via reference compounds; ${ }^{2}$ highlights peaks that were shown to be double peaks in a part of the accessions (characteristics of the overlain peak are indicated below the respective line). Identification literature [11-13,26,31,36-38].

\begin{tabular}{|c|c|c|c|c|c|c|}
\hline Peak & $\underset{\text { (min) }}{\mathrm{Rt}}$ & $\begin{array}{c}\mathrm{M}-\mathrm{H}]^{-} \\
(\mathrm{m} / \mathrm{z})\end{array}$ & $\underset{(\mathrm{m} / \mathrm{z})}{\mathrm{MS}}$ & Proposed Compound & Literature & $\underset{\%}{\text { Rel. Area }}$ \\
\hline 1 & 10.1 & 1083 & $301 / 541$ & Punicalagin, isomer $1^{1}$ & {$[11,13,26,31,36]$} & $0-16 \%$ \\
\hline 2 & 14.4 & 1083 & $301 / 541$ & Punicalagin, isomer $2^{1}$ & {$[11,13,26,31,36]$} & $0-19 \%$ \\
\hline 3 & 14.8 & 1251 & $541 / 603$ & Punicalagin gallate, isomer 1 & {$[11,26,31,36]$} & $0-10 \%$ \\
\hline 4 & 22.1 & 1251 & $541 / 603$ & Punicalagin gallate, isomer 2 & {$[11,26,31,36]$} & $0-14 \%$ \\
\hline 5 & 32.1 & 479 & 316 & M-3-O-galactoside & {$[11,12,26,31,37,38]$} & $0-47 \%$ \\
\hline 6 & 32.8 & 479 & 316 & M-3-O-glucoside & {$[11-13,26,31,37,38]$} & $0-6 \%$ \\
\hline 7 & 37.3 & 449 & 316 & M-O-xyloside and/or m-3-O-arabinoside ${ }^{2}$ & {$[26,31,37,38]$} & $0-50 \%$ \\
\hline 8 & 38.4 & 463 & 316 & M-3-O-rhamnoside (myricitrin) ${ }^{1}$ & {$[11-13,26,31,37,38]$} & $5-83 \%$ \\
\hline \multirow[t]{2}{*}{9} & 39.3 & 609 & 301 & Q-3-O-rutinoside (rutin) ${ }^{1,2}$ & {$[11-13,26,31,37,38]$} & $0-17 \%$ \\
\hline & & 521 & 316 & Putative m-derivative, ev. an artifact & & \\
\hline 10 & 40.0 & 463 & 301 & Q-3-O-galactoside (hyperoside) ${ }^{1}$ & {$[26,31,37,38]$} & $0-12 \%$ \\
\hline 11 & 40.8 & 463 & 301 & Q-3-O-glucoside (isoquercetin) ${ }^{1}$ & {$[11,13,26,31,36-38]$} & $0-3 \%$ \\
\hline 12 & 46.0 & 433 & 301 & Q-O-xyloside and/or q-3-O-arabinoside ${ }^{2}$ & {$[11-13,26,31,36-38]$} & $0-9 \%$ \\
\hline 13 & 48.9 & 447 & 301 & Q-3-O-rhamnoside (quercitrin) ${ }^{1}$ & {$[11-13,26,31,37,38]$} & $0-35 \%$ \\
\hline
\end{tabular}

Among the minor compounds, the presence of further myricetin glycosides (one $\mathrm{m}$-O-rhamnoside-O-hexoside, one putative breakdown product of $\mathrm{m}$-3-O-rhamnoside), quercetin glycosides (one q-pentoside, q-3-O-rutinoside-7-O-hexoside and/or q-3-O -(2'caffeoyl)-rutinoside and one further q-derivative) and ellagitannins (cornusiin $B$ isomers, bis-hexahydroxydiphenoyl (HHDP)-glucose or pedunculagin, galloyl-HHDP-glucoside, probably 7-xyloside ellagic acid) was indicated (data not shown). Further minor compounds were tentatively identified as kaempferol derivatives (k-3-O-galactoside or -glucoside, kdiglucosides(s) or tiliroside isomers and one further putative k-derivative), flavanols 
((epi)gallocatechin dimer or prodelphinidin B4, (epi)catechin, (epi)gallocatechin trimer) and a propiophenone derivative (3,4'-dihydroxypropiophenone-3- $\beta$-D-glucoside).

To visualize the incidence and variability of the major compounds, the peak areas of the four main myricetin glycosides (m-3-O-galactoside, m-3-O-glucoside, $\mathrm{m}$-O-xyloside or m3-O-arabinoside, m-3-O-rhamnoside), the five main quercetin glycosides (q-3-O-rutinoside, q-3-O-galactoside, q-3-O-glucoside, q-O-xyloside or q-3-O-arabinoside, q-3-O-rhamnoside) and the two punicalagin derivatives (punicalagin and punicalagin gallate) were quantified (equivalent to the respective major compound m-3-O-rhamnoside, q-3-O-rhamnoside and punicalagin), cumulated and plotted (Figure 1).
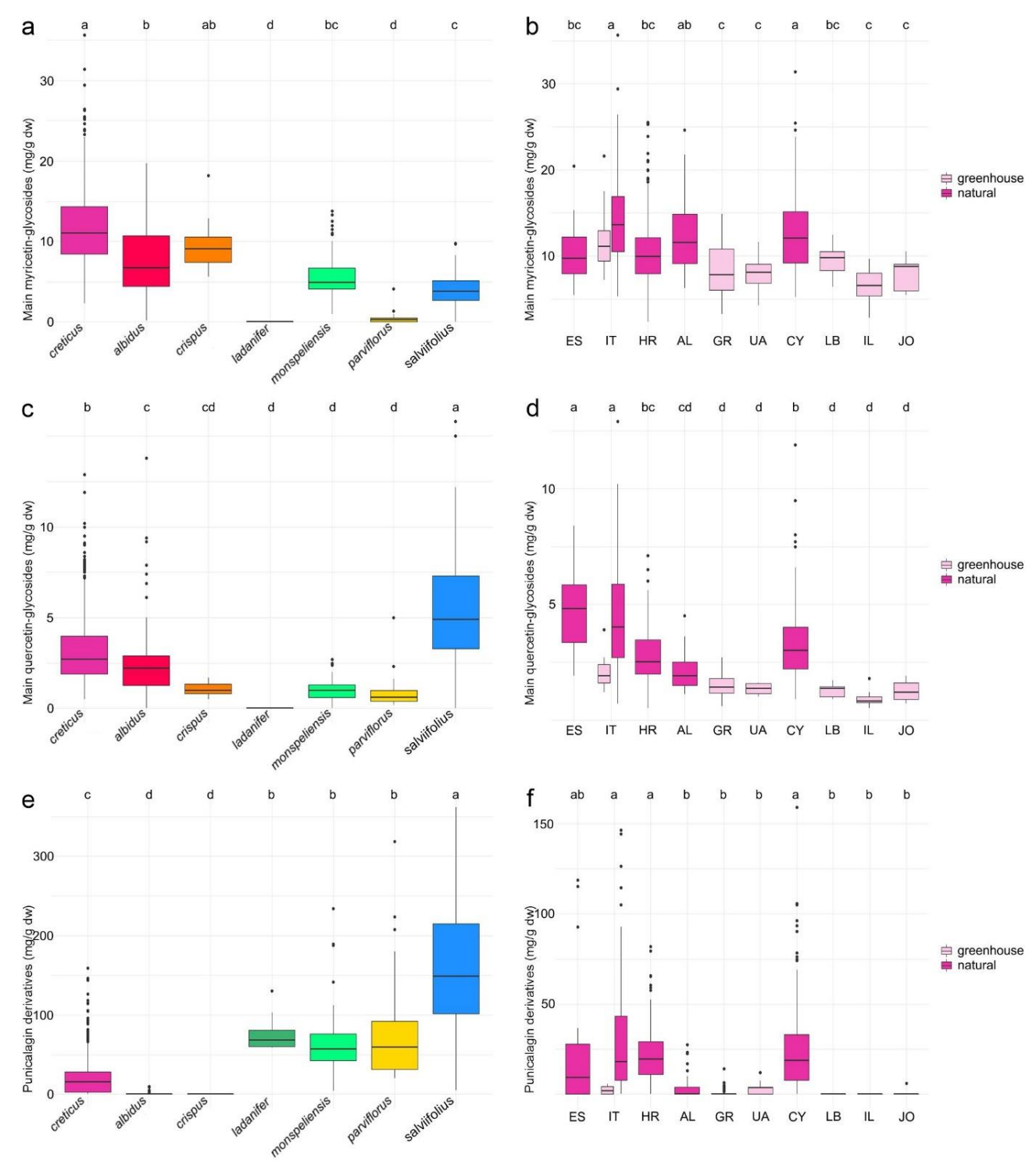

Figure 1. Comparison of (a) myricetin glycoside amounts (mg/g dry wt), (c) quercetin glycoside amounts (mg/g dry wt) and (e) amount of punicalagin derivatives (mg/g dry wt) of C. creticus, C. albidus, C. crispus, C. ladanifer, C. monspeliensis, C. parviflorus and $C$. salviifolius. Species with the same letter on top do not differ significantly from each other (groups were determined by Tukey honestly significant difference (HSD) test, alpha $=0.005)$. Comparison of $(\mathbf{b})$ myricetin glycoside amounts (mg/g dry $\mathrm{wt})$, (d) quercetin glycoside amounts $(\mathrm{mg} / \mathrm{g}$ dry wt) and (f) ellagitannin amounts $(\mathrm{mg} / \mathrm{g}$ dry wt) of $C$. creticus populations originating from Spain to Jordan. Colors determine natural (pink) or greenhouse origin (light pink) of plant material. Countries with the same letter on top do not differ significantly from each other (groups were determined by Tukey HSD test, alpha = 0.005). $\mathrm{ES}=$ Spain (three populations), IT = Italy (two cultivated populations and eleven natural populations), $\mathrm{HR}=\mathrm{Croatia}$ (16 populations), $\mathrm{AL}=$ Albania (four populations), $\mathrm{GR}=$ Greece (four populations), $\mathrm{UA}=$ Ukraine (one population), $\mathrm{CY}=$ Cyprus (28 populations), LB = Lebanon (one population), $\mathrm{IL}=$ Israel (one population), JO = Jordan (one population). 


\subsubsection{Myricetin Glycosides}

The overall highest contents of myricetin derivatives were present in the three flavonolrich, purple-flowering species (C. creticus, C. albidus and C. crispus), whereas the white (C. ladanifer, C. monspeliensis, C. salviifolius)- or pink (C. parviflorus)-flowering species exhibited lower amounts or were devoid of some of these compounds (Figure 1a). With a median content of $11 \mathrm{mg} / \mathrm{g}$ dry wt, C. creticus differed significantly from C. albidus $(7 \mathrm{mg} / \mathrm{g}$ dry wt), C. salviifolius (4 $\mathrm{mg} / \mathrm{g}$ dry $\mathrm{wt}$ ) and the pair C. ladanifer and C. parviflorus ( $<\mathrm{LOD}$ and $0.3 \mathrm{mg} / \mathrm{g}$ dry wt). Within the purple-flowered species, C. crispus $(9 \mathrm{mg} / \mathrm{g}$ dry wt) could not be differentiated from C. creticus and C. albidus. Cistus monspeliensis, with $5 \mathrm{mg} / \mathrm{g}$ dry $w \mathrm{t}$, the white-flowered species richest in myricetin glycosides, occupied a central position between $C$. albidus and C. salviifolius. Within the three purple-flowering species, m-3-O-rhamnoside was the main myricetin glycoside and main compound (up to $83 \%$ relative peak area percentage in C. albidus; data not shown). In rare cases, $\mathrm{m}$-3-O-galactoside was higher than m-3-O-rhamnoside (up to $47 \%$ in an Albanian accession of C. creticus; data not shown). Within the white- or the pink-flowering species, a predominance of $\mathrm{m}$-3-O-galactoside or $\mathrm{m}-\mathrm{O}$-xyloside was more frequent or the rule. Within $\mathrm{C}$. parviflorus and C. salviifolius, m-3-O-rhamnoside was generally low or even not present (Table S1, Supplementary Material).

Within C. creticus, the highest amounts of myricetin glycosides were detected in Italy and Cyprus (median content of 13 and $12 \mathrm{mg} / \mathrm{g}$ dry wt, respectively; Figure 1b). Populations deriving from these two countries differed significantly from populations originating from Greece, Ukraine, Israel and Jordan grown in the greenhouse (median contents between 5 and $8 \mathrm{mg} / \mathrm{g}$ dry wt) that exhibited the overall lowest amounts of myricetin glycosides. Wild populations of Albania ( $12 \mathrm{mg} / \mathrm{g}$ dry wt) were not significantly different from the populations richest in myricetin glycosides. Wild populations from Spain and Croatia (both $10 \mathrm{mg} / \mathrm{g}$ dry wt) and the Lebanese greenhouse population $(10 \mathrm{mg} / \mathrm{g}$ dry $\mathrm{wt}$ ) were not significantly different from populations poor in myricetin glycosides.

\subsubsection{Quercetin Glycosides}

With a median content of $5 \mathrm{mg} / \mathrm{g}$ dry wt, C. salviifolius was the species with the highest content of quercetin derivatives (Figure 1c). Significant differences were detected between C. salviifolius, C. creticus ( $3 \mathrm{mg} / \mathrm{g}$ dry wt), C. albidus $(2 \mathrm{mg} / \mathrm{g}$ dry $\mathrm{wt})$ and the three species comparatively low in or completely lacking quercetin glycosides (C. ladanifer, C. monspeliensis and C. parviflorus with contents between 0 and $1 \mathrm{mg} / \mathrm{g}$ dry wt). Cistus crispus ( $1 \mathrm{mg} / \mathrm{g}$ dry wt) took a central position between C. albidus and the three whiteflowering species low in quercetin glycosides. The extraordinary $C$. salviifolius was the sole species with quercetin glycosides as the predominant flavonol compound family. In the flavonol-rich accessions of purple-flowering C. creticus, C. albidus and C. crispus, the contents of quercetin glycosides were usually conspicuously lower than those of the myricetin glycosides. Within C. monspeliensis and C. parviflorus, the predominance of the myricetin glycosides was not that distinctive. Cistus ladanifer lacked both myricetin and quercetin glycosides. In C. creticus and C. albidus, q-3-O-rhamnoside was often the main quercetin glycoside (up to $34 \%$ and $80 \%$ relative peak area percentage; data not shown). However, the predominance of the quercetin rhamnoside was not as pronounced as that of the myricetin rhamnoside (see above). Some accessions even showed q-3-O-rutinoside or q-3-O-galactoside as the main quercetin glycosides (about $20 \%$ of the $C$. creticus accessions, about $2 \%$ of the $\mathrm{C}$. albidus accessions; data not shown). Within $\mathrm{C}$. crispus, q-3-O-galactoside was the main quercetin glycoside. Within $\mathrm{C}$. monspeliensis, q-3-O-galactoside, q-O-xyloside and q-3-O-rhamnoside were present in higher amounts. Within $C$. parviflorus and $C$. salviifolius, q-3-O-rhamnoside was only present in trace amounts and q-3-O-galactoside and q-O-xyloside (and/or or q-3-O-arabinoside) were the main quercetin glycosides (Table S1, Supplementary Material).

Within C. creticus, the overall highest amounts of quercetin glycosides were present in populations of Spain and Italy (median contents of 5 and $4 \mathrm{mg} / \mathrm{g}$ dry wt; Figure 1d). 
Both can clearly be differentiated from populations of Cyprus ( $3 \mathrm{mg} / \mathrm{g}$ dry wt) and the greenhouse populations from Greece, Ukraine and the Near East (all around $1 \mathrm{mg} / \mathrm{g}$ dry wt). The populations from Croatia and Albania (both with a median content around $2 \mathrm{mg} / \mathrm{g}$ dry wt) were not clearly differentiated.

\subsubsection{Punicalagin Derivatives}

The overall highest amounts of punicalagin and punicalagin gallate were present in the also here outstanding C. salviifolius (median content of $149 \mathrm{mg} / \mathrm{g}$ dry wt). Cistus salviifolius differed significantly from the further three white- or pink-flowering species (C. ladanifer, C. monspeliensis and C. parviflorus, with median contents between 57 and $68 \mathrm{mg} / \mathrm{g}$ dry wt). Cistus salviifolius also differed from C. creticus (15 mg/g dry wt) and the other two purple-flowering species (C. albidus and C. crispus), who exhibited no or solely traces of punicalagin and punicalagin gallate (Figure 1e). Cistus creticus was the only purple-flowered species with accessions exhibiting noteworthy amounts of punicalagin derivatives. Higher proportions of punicalagin and punicalagin gallate were usually also detected within C. ladanifer. Cistus monspeliensis, C. parviflorus and C. salviifolius were rich in punicalagin but the two punicalagin gallate isomers were not present in higher amounts (except a smaller number of single accessions from the whole distribution area) (Table S1, Supplementary Material). Beside the two punicalagin gallate peaks evaluated, however, the chromatograms of white- and whitish-pink-flowering species exhibited two additional, very conspicuous peaks with a clear punicalagin gallate signature indicating the presence of two further isomers that are specific for the white- and whitish-pink-flowered clade.

Within C. creticus, the highest proportions of punicalagin and punicalagin gallate were detected in the Italian, Croatian and Cypriot populations (median contents between 16 and $19 \mathrm{mg} / \mathrm{g}$ dry wt, Figure 1f). Populations from these three countries differed significantly from those of Albania, Greece, Ukraine and the Near East (all with median contents between 0 and $4 \mathrm{mg} / \mathrm{g}$ dry wt). The populations from Spain $(9 \mathrm{mg} / \mathrm{g}$ dry wt) were between punicalagin derivative-rich and -poor C. creticus populations. Regarding the two evaluated ellagitannins, C. creticus was extremely variable. About $20 \%$ of the C. creticus samples were devoid of punicalagin and punicalagin gallate (more specifically amounts below our calculated LOD of $0.2 \mu \mathrm{g} / \mu \mathrm{L}$ ). Some accessions of $C$. creticus contained lower amounts of punicalagin but no detectable amounts of punicalagin gallate, and some exhibited lower amounts of both. Single accessions reached amounts comparable to those extracted from the more ellagitannin-rich species. A high diversity was observed especially in Italy where the punicalagin derivative contents of C. creticus ranged from $<$ LOD to $147 \mathrm{mg} / \mathrm{g}$ dry wt.

\subsection{Sample Classification}

A principal component analysis (PCA) was performed by using composition data of the 13 major peaks (relative peak area percentages; Figure 2a). The first two dimensions explained about $59 \%$ of the variance. Principal component 1 (41\%) differentiated purplefrom white- or whitish-pink-flowering species. The most important variables responsible for this differentiation include the two flavonols myricetin- and quercetin-3-O-rhamnoside as well as the four peaks representing the punicalagin derivatives (Figure 2b). Moreover, PC1 separated C. monspeliensis from the three other white- or pink-flowering species, with some $\mathrm{m}$-glycosides as discriminating variables. Within the purple-flowered clade, principal component 2 (18\% of the variation) distinguished C. creticus and C. albidus from C. crispus. The most influential variable here was m-3-O-galactoside. Within the white- and whitishpink-flowered clade, $\mathrm{PC} 2$ provided only marginal distinguishing power. The separation of $C$. ladanifer individuals along a straight line reflected the complete lack of nine of the thirteen included peaks (all nine myricetin and quercetin glycosides). With a closer look at the $C$. creticus populations, no conspicuous intraspecific differentiation was obvious (Figure 2c). According to principal component $1(33 \%)$, all the cultivated populations clustered closely together on the left margin based on the predominance of q- and m-3O-rhamnoside (Figure 2d). Principal component 2 (28\%) provided no noticeable discrim- 
ination between cultivated and wild populations or populations of different countries. However, the group midpoints of the geographically distant Albanian and Spanish populations clustered more closely to each other (and to the group midpoints of the cultivated populations) than to their geographically closer populations.

a

a Individuals - PCA
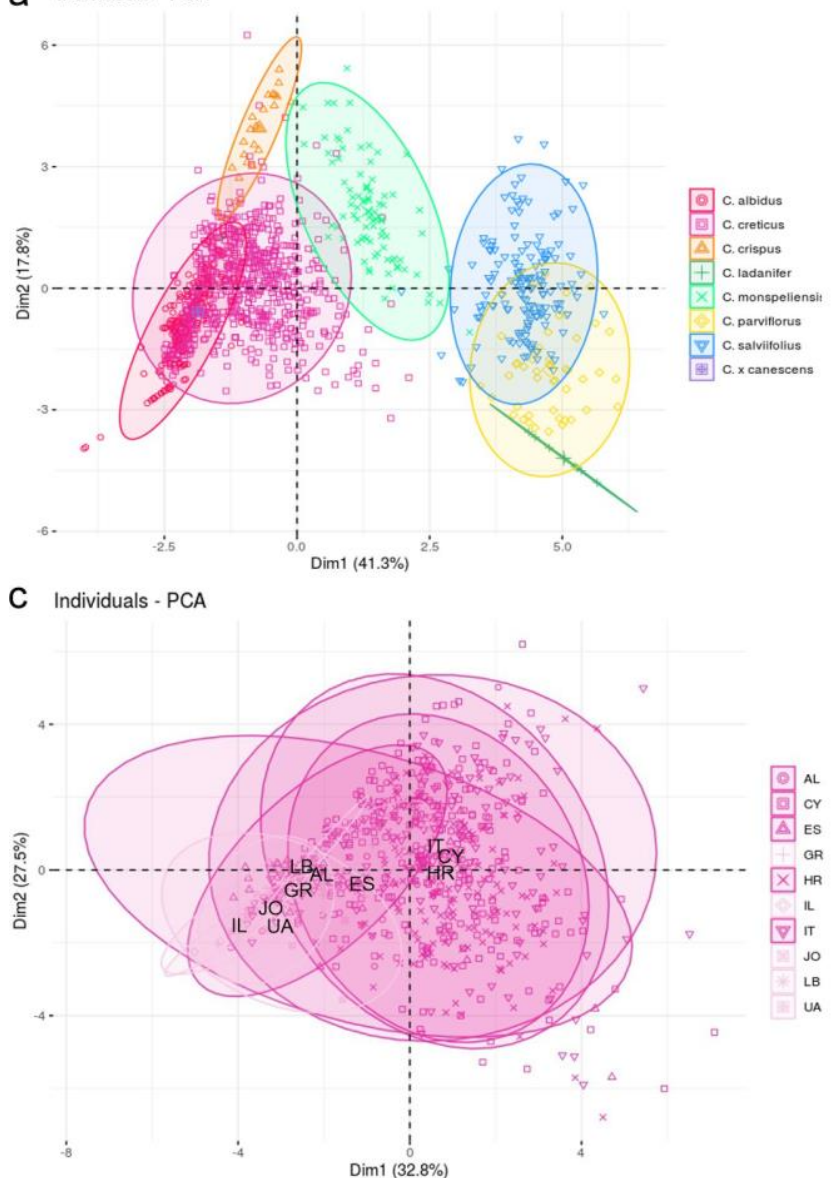

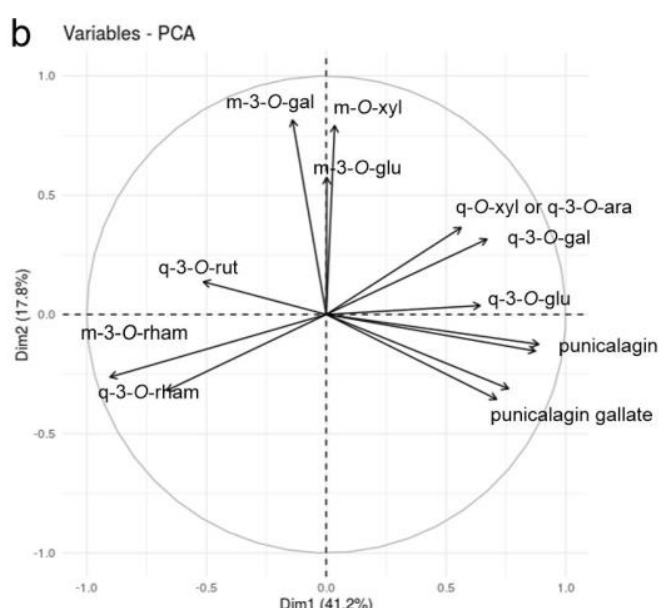

d Variables - PCA

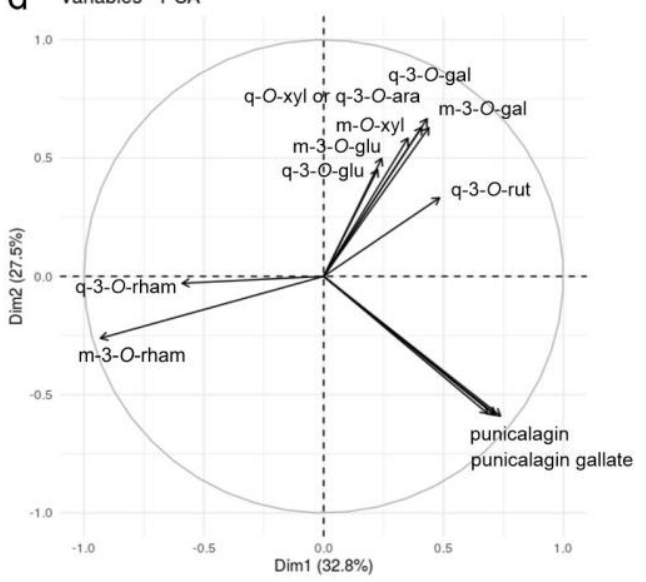

Figure 2. Principal component analysis (PCA) and corresponding factor loadings. (a) Plot of the first two dimensions (dim) from PCA performed over all seven species and the eleven evaluated compounds (relative area percent). In (b), the influence of each included variable is indicated. (c) Plot of first and second principal components from PCA performed over all sampled C. creticus populations (countrywise combined, group midpoints were accentuated) and the eleven main compounds used for quantification (relative area percent). (d) Factor loadings of the thirteen variables included. The percentage of variance explained by each dimension is indicated in parenthesis. Colors indicate either species affiliation (2a) or differentiate wild (pink) and cultivated (light pink) populations (2c). ES = Spain (three populations), IT = Italy (two cultivated populations and eleven natural populations), $\mathrm{HR}=$ Croatia (16 populations), AL = Albania (four populations), GR = Greece (four populations), UA = Ukraine (one population), $\mathrm{CY}=$ Cyprus (28 populations), $\mathrm{LB}=$ Lebanon (one population), $\mathrm{IL}=$ Israel (one population), JO = Jordan (one population).

\subsection{Extract Composition and Classification of Trade Samples}

Fifteen trade samples of coarse-cut Cistus products (one trade sample was labeled as C. creticus, twelve as C. incanus and two samples as Cistus sp.; Table 2) were purchased from different suppliers to compare their compound composition with that of the wild and greenhouse populations. The four main myricetin glycosides summed up ranged between 1 and $10 \mathrm{mg} / \mathrm{g}$ dry $\mathrm{wt}$, that of the five main quercetin glycosides between $<$ LOD and $3 \mathrm{mg} / \mathrm{g}$ dry wt and that of the two punicalagin derivatives between $<\mathrm{LOD}$ and $161 \mathrm{mg} / \mathrm{g}$ dry wt (Table 2). As the peak patterns of the chromatograms of the trade samples appeared rather heterogeneous and exhibited attributes characteristic for different species, we refrained 
from including them as one sample group in the primary statistical analysis and plots. The heterogeneity of the trade samples was well demonstrated when included in the PCA analysis (Figure S2, Supplementary Material). Only five samples grouped within or at least close to samples of $C$. creticus, whereas the composition of the other trade samples corresponded more to that of white-flowering species (C. monspeliensis and C. salviifolius).

Table 2. Designation and geographical origin (when indicated) of the 15 commercial trade samples purchased from different pharmacies ${ }^{1}$ or health retailers ${ }^{2}(\mathrm{kbA}=$ controlled organic cultivation) and their contents of punicalagin derivatives and myricetin and quercetin glycosides ( $\mathrm{mg} / \mathrm{g}$ dry weight).

\begin{tabular}{|c|c|c|c|c|c|c|}
\hline Sample & Declaration & Origin & P-Derivatives & M-Glycosides & Q-Glycosides & $\begin{array}{c}\text { Tentative } \\
\text { Identification }\end{array}$ \\
\hline & & & (mg/g dry wt) & $(\mathrm{mg} / \mathrm{g}$ dry wt) & (mg/g dry wt) & (via HPLC profile) \\
\hline $\mathrm{CHP}^{1}{ }^{1}$ & Cistus sp. & TR & 161.2 & 4.8 & 2.4 & White-flowered species \\
\hline $\mathrm{CHPO2}^{1}$ & C. incanus L. & GR & $<\mathrm{LOD}$ & 4.0 & 0.8 & C. creticus \\
\hline $\mathrm{CHP03}^{1}$ & C. incanus & - & 16.8 & 10.0 & 2.5 & C. creticus \\
\hline $\mathrm{CHPO}^{1}$ & C. incanus L. & GR & $<\mathrm{LOD}$ & 0.7 & 0.2 & C. creticus \\
\hline $\mathrm{CHP}^{2} 5^{2}$ & C. incanus & - & 81.1 & 4.1 & 1.5 & White-flowered species \\
\hline $\mathrm{CHPO6}^{2}$ & C. incanus $\mathrm{kbA}$ & - & 33.1 & 0.5 & 0.2 & White-flowered species \\
\hline $\mathrm{CHP}^{2}{ }^{2}$ & C. incanus $\mathrm{kbA}$ & $\mathrm{CY}$ & 85.3 & 8.4 & 2.3 & C. creticus \\
\hline $\mathrm{CHP}^{2}{ }^{2}$ & C. incanus & $\mathrm{TR}$ & 22.1 & 8.6 & 2.0 & $\begin{array}{c}\text { C. creticus } \\
\text { (adulterated?) }\end{array}$ \\
\hline $\mathrm{CHP}^{2}{ }^{2}$ & C. incanus & - & 50.7 & 5.9 & 1.7 & White-flowered species \\
\hline $\mathrm{CHP} 10^{2}$ & C. incanus & - & 61.5 & 4.0 & 1.3 & White-flowered species \\
\hline CHP11 ${ }^{2}$ & C. incanus & - & 79.6 & 3.5 & 1.1 & White-flowered species \\
\hline CHP12 ${ }^{2}$ & C. creticus & - & 123.3 & 3.3 & 3.0 & White-flowered species \\
\hline $\mathrm{CHP} 13^{2}$ & C. incanus $\mathrm{kbA}$ & $\mathrm{TR}$ & 66.0 & 3.2 & 1.4 & White-flowered species \\
\hline $\mathrm{CHP} 14^{2}$ & C. incanus & - & 82.9 & 5.1 & 2.7 & White-flowered species \\
\hline $\mathrm{CHP} 15^{2}$ & Cistus sp. & TR & 29.4 & 6.5 & 1.5 & $\begin{array}{c}\text { C. creticus } \\
\text { (adulterated?) }\end{array}$ \\
\hline
\end{tabular}

\subsection{Antioxidant Activity and Total Phenolics}

A DPPH radical scavenging assay was used to characterize the antioxidant activity of the Cistus plant samples. The highest antioxidant activities were present in C. ladanifer (median antioxidant capacity of 301 milligram Trolox equivalents per gram dry weight) and C. salviifolius (261 mg te/g dry wt; Figure 3a). Both differed significantly from C. albidus (142 mg te/g dry wt) with the overall lowest antioxidative activity. Cistus crispus (201 mg te/g dry wt) was not clearly differentiated from the two species with the highest activities. Cistus monspeliensis (171 $\mathrm{mg}$ te/g dry wt), C. creticus (166 mg te/g dry wt) and C. parviflorus (147 $\mathrm{mg}$ te/g dry wt) were not different from C. albidus, the species with the lowest antioxidant activity.

Within C. creticus, the highest antioxidant capacity was present in the Albanian populations (median antioxidant capacity of $232 \mathrm{mg}$ te/g dry wt; Figure 3b). The populations from Albania were significantly different from greenhouse populations of closely located Greece that exhibited the lowest antioxidant activity (115 $\mathrm{mg}$ te/g dry wt). The Lebanese (230 mg te/g dry wt) and Israeli (198 mg te/g dry wt) populations could not be differentiated from the Albanian populations. The wild populations from Italy (174 $\mathrm{mg}$ te/g dry wt), Cyprus (170 mg te/g dry wt), Spain (164 mg te/g dry wt) and Croatia (160 mg te/g dry wt) as well as the greenhouse populations originating from Israel (198 $\mathrm{mg}$ te/g dry $w t)$, Jordan (170 mg te/g dry wt) and Ukraine (139 $\mathrm{mg}$ te/g dry wt), were not significantly different from the Greek populations with the lowest antioxidative capacity. 

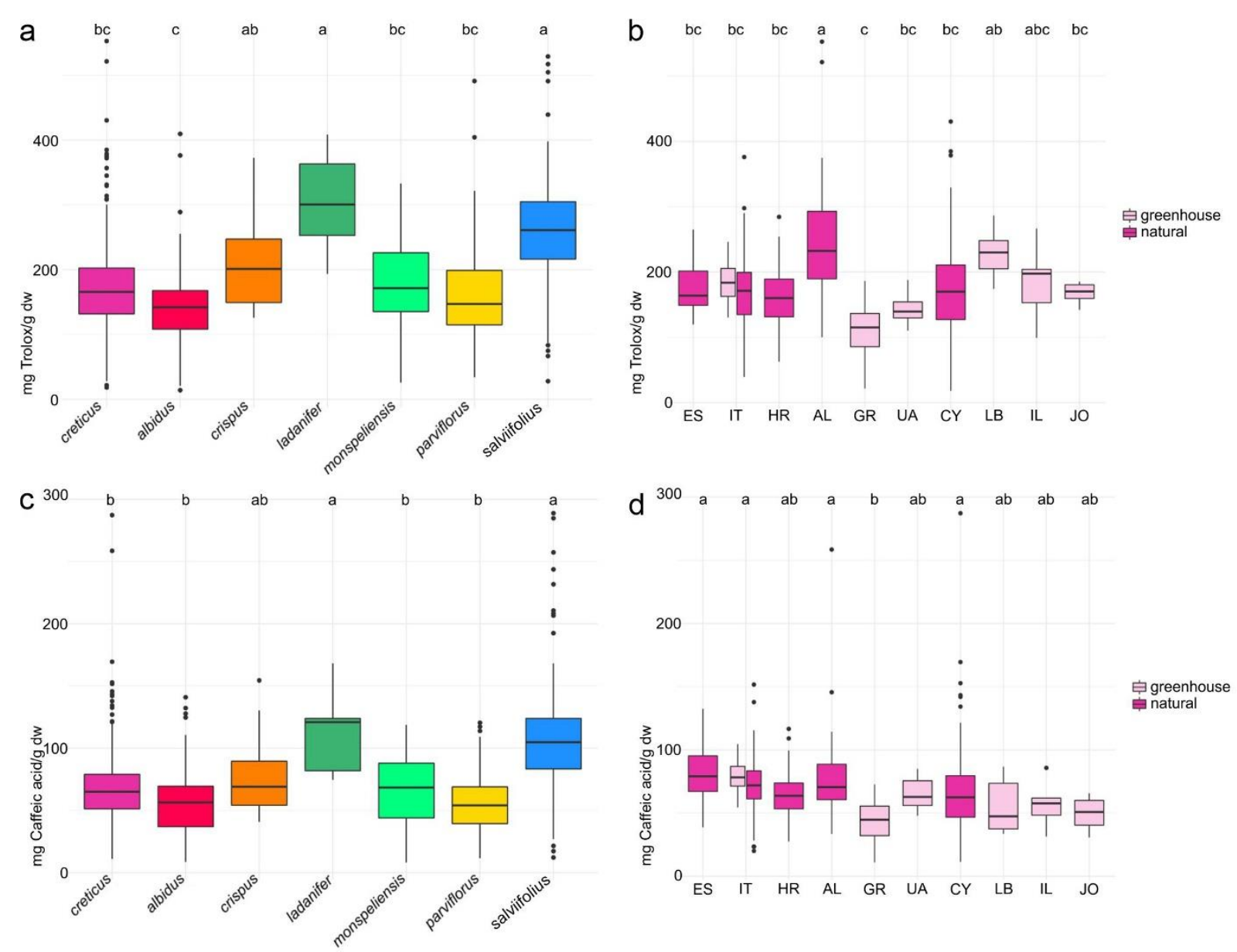

Figure 3. Comparison of (a) 2,2-diphenyl-1-picrylhydrazyl (DPPH) radical scavenging activity (mg te/g dry wt) and (c) total phenolic content (mg cae/g dry wt) of C. creticus, C. albidus, C. crispus, C. ladanifer, C. monspeliensis, C. parviflorus and C. salviifolius. Species with the same letter on top do not differ significantly from each other (groups were determined by Tukey HSD test, alpha $=0.005)$. Comparison of $(\mathbf{b})$ DPPH radical scavenging activity $(\mathrm{mg}$ te/g dry wt) and $(\mathbf{d})$ total phenolic content (mg cae/g dry wt) of C. creticus populations originating from Spain to Jordan. Colors determine either species or natural (pink) or greenhouse origin (light pink) of the analyzed plant material. Countries with the same letter on top do not differ significantly from each other (groups were determined by Tukey honestly significant difference (HSD) test, alpha $=0.005)$. ES = Spain (three populations), IT = Italy (two cultivated populations and eleven natural populations), HR = Croatia (16 populations), $\mathrm{AL}=$ Albania (four populations), GR = Greece (four populations), UA = Ukraine (one population), $\mathrm{CY}=$ Cyprus (28 populations), LB = Lebanon (one population), $\mathrm{IL}=$ Israel (one population), JO = Jordan (one population).

The total phenolic content of Cistus plant samples was evaluated spectrophotometrically using caffeic acid as the standard. The highest contents of phenolic compounds were again present within C. ladanifer (121 milligram caffeic acid equivalents per gram dry weight) and C. salviifolius (105 $\mathrm{mg}$ cae/g dry wt) that can clearly be differentiated from C. monspeliensis (68 $\mathrm{mg}$ cae/g dry wt), C. creticus (65 mg cae/g dry wt), C. parviflorus (54 mg cae/g dry wt) and C. albidus (56 mg cae/g dry wt; Figure 3c). Cistus crispus (69 mg cae/g dry wt) could not clearly be differentiated from both the species with higher and the species with comparatively low contents of total phenolics.

Within C. creticus, the Albanian populations (71 mg cae/g dry wt), together with Spanish (79 mg cae/g dry wt), Italian (73 mg cae/g dry wt) and Cypriot populations (62 mg cae/g dry wt; Figure 3d), exhibited the highest median contents of phenolic compounds. They were statistically different from the greenhouse populations from Greece (45 mg cae/g dry wt) with the overall lowest contents of total phenolic compounds. Populations originating from Croatia (64 mg cae/g dry wt), the Ukraine (63 mg cae/g dry wt) 
and the Near East (median contents of total phenolics between 47 and $58 \mathrm{mg}$ cae/g dry $w t)$ were not significantly different from both. Similar to the results from the DPPH radical scavenging assay, no significant influence of origin (from wild or cultivated populations) was detectable (data not shown).

When comparing the plots describing DPPH and phenolic content, a high similarity between the patterns of Figure $3 a, b$ and of Figure $3 c, d$ became obvious, indicating a similar variability of both parameters between Cistus species and C. creticus populations from different countries. This was statistically confirmed by a strong positive correlation $(r=0.77)$ between antioxidant capacity and phenolic content (Table S2, Supplementary Material). However, there was only a positive correlation between antioxidative activity/total phenolics and the summed content of punicalagin and punicalagin gallate $(r=0.47 / 0.43)$ and only a rather weak positive correlation between antioxidative activity/total phenolics and the summed content of quercetin glycosides $(r=0.27 / 0.34)$. Myricetin glycosides do not participate much in antioxidative activity $(r=-0.05)$.

\section{Discussion}

Plant material of seven Cistus species from 13 Mediterranean countries was sampled, aiming to provide a primary inventory of natural flavonoid variability within C. creticus and to allow a direct comparison of qualitative and quantitative extract composition with that of different, partly sympatric Cistus species.

To optimize sample preparation, initial experiments were performed to assess the influence of different sample weights and extraction times and to compare the composition of aqueous (based on the protocol of [11]) and hydromethanolic extracts (as used, e.g., by $[32,39])$. The different solvent extracts exhibited highly comparable peak patterns but different quantitative characteristics (Figure S3, Supplementary Material). Compared to hydromethanolic extracts $(50 \%)$, pure water extracts exhibited higher amounts of the two punicalagin derivatives considered in the work (5 to 30\%) and lower amounts of the main flavonoid compounds (20 to $40 \%$ ). Based on the primary results, we decided on deionized water as the extraction medium because of the compositional similarity of water extracts to Cistus tea preparations, the most common pharmaceutical form of use. One further, pragmatic argument was the more safe and sustainable extraction procedure for the high number of samples expected. The here presented values quantifying total phenolics and antioxidant capacity might be, by trend, lower than those with hydroethanolic or hydromethanolic extracts (see also [33], who comparatively discussed characteristics of aqueous and hydroethanolic C. incanus extracts).

Qualitatively, chromatograms of all the seven Cistus species exhibited quite stable and, for the firmly trained eye, very distinguishing peak patterns (Figure S1, Supplementary Material). Quantitatively, a wide range of variation was observed. Polyphenols are thought to be important factors for plants' ability to cope with difficult environmental conditions and are supposed to aid their persistence in extreme habitats. As highly responsive to specific habitat conditions, polyphenolic compound levels and patterns can vary significantly within a species (e.g., [40-43]). The authors of [44] observed a moderate positive influence of temperature and solar irradiance on the total phenolic content of $C$. incanus, specifically on the content of quercetin and tannin derivatives. The authors of [45] described a suite of genes regulating flavonoid biosynthesis and transport to be largely overexpressed in sun-adapted leaves of $C$. incanus and reported a light-induced accumulation of myricetin and quercetin glycosides. Backed up by these results, it can be hypothesized that the lower compound levels of the potted populations can be primarily ascribed to latitude-related effects such as lower mean solar irradiance and mean temperatures during the year. The wild Cistus plants were sampled along a wide geographical gradient and from various habitats. Each individual plant was exposed to specific environmental conditions and responded to them in an individual way. To overcome this variability and to obtain statistical significance, we collected plant material from a high number of widespread populations and analyzed, in most cases, at least ten individual plants per population. To minimize 
effects related to plant development or season [33,44], plants exhibiting a comparable phenological stage (full to ending bloom) were harvested. Based on this carefully collected and extensive sample set, persistent overall tendencies were found.

Extracts of $C$. creticus were usually characterized by punicalagin as the main compound in the "ellagitannin-half" and m-3-O-rhamnoside (in rare cases m-3-O-galactoside) as the main compound in the "flavonol-half" of the chromatogram. In our sample set, about $80 \%$ of the C. creticus plants exhibited both flavonol glycosides and punicalagin derivates. About $20 \%$ of our C. creticus samples were devoid of punicalagin and punicalagin gallate (more specifically amounts $<\mathrm{LOD}$ of $0.2 \mu \mathrm{g} / \mu \mathrm{L}$ ). This nearly pure flavonol variant was detected from Spain to the Near East, with higher abundance in the most western (37\% in Spain) and more easternmost populations (40\% in Albania and the Ukraine, $75 \%$ in Greece, up to $100 \%$ in populations of the Near East) and a conspicuously lesser frequency in Italy $(9 \%)$, Croatia ( $8 \%$ ) and Cyprus (12\%). The rarer flavonol chemovariant relates C. creticus to its close relatives $C$. albidus and C. crispus and seems to be a specific characteristic of the purple-flowered clade, as was already postulated by [11]. Compared to C. creticus, the chromatogram characteristics of $C$. albidus appeared to be more stable, with the flavonol variant as the predominant one ( $87 \%$ of the samples) and few individual plants exhibiting comparatively small amounts of punicalagin derivatives. Within the two populations of C. crispus, solely the flavonol variant was detected. The frequent chemovariant characterized by the presence of flavonol glycosides and punicalagin derivatives relates purple-flowered C. creticus to the white- or whitish-pink-flowered species $C$. ladanifer, C. monspeliensis, C. parviflorus and C. salviifolius. Compared to C. creticus, the white- or whitish-pink-flowering species usually contained higher percentages and amounts of punicalagin and, in many cases, also of punicalagin gallate. Moreover, within the white- and pink-flowered species, two additional punicalagin gallate isomers were prominently present, indicating cladeand species-specific peculiarities in the respective biosynthetic pathway. In the white- or whitish-pink-flowering species, flavonol compounds were usually of less importance than in the purple-flowering ones. The one population of C. ladanifer completely lacked the myricetin and quercetin glycosides and both compound families were scarcely present within C. parviflorus. Somehow, higher percentages of flavonols were detected within C. monspeliensis and C. salviifolius, the species that was exceptional regarding its variability and partly outstanding high content of quercetin glycosides. Regarding chemotype composition, our results resembled, in principle, previous results described from single or few samples of Spanish C. creticus, C. albidus, C. crispus, C. ladanifer, C. salviifolius and C. monspeliensis [11] and Italian C. creticus [12-14,44]. One further sample of Portuguese C. ladanifer exhibited, contrary to [11] and our results, also smaller amounts of quercetin glycosides [36].

The first two dimensions of the principal component analysis visualize well the outcomes discussed above and earlier hypotheses that were based on the analysis of single or few extracts of ten different Cistus species [11]. According to their polyphenolic profiles, the purple-flowered clade (subgenus Cistus) could be well separated from the white- and whitish-pink-flowered clade (all four species here representing subgenus Leucocistus). This differentiation was mainly based on the presence and proportions of punicalagin, punicalagin gallate and m- and q-3-O-rhamnoside, but also further myricetin and quercetin derivatives were involved. Within the purple-flowered clade, C. crispus was clearly distinct, whereas C. creticus and C. albidus were not differentiated from each other. These results resembled earlier findings based on DNA sequence and pollen analysis that postulated a close evolutionary relationship of $C$. creticus and $C$. albidus with $C$. crispus as a more distantly related sister taxon [1,2]. Obviously, the separation of the purple-flowered and the white- and whitish-pink-flowered clade was not entirely perfect, mainly due to the many Italian, Croatian and Cypriot accessions of $C$. creticus that exhibited comparatively high percentages of punicalagin derivatives and tended towards subgenus Leucocistus. The unequal geographical distribution of plants rich in punicalagin derivatives within C. creticus was also lightly indicated in the PCA plots. The two group midpoints repre- 
senting C. creticus from geographically distinct Spain and Albania, respectively, clustered more closely to each other than to the group midpoints representing the accessions from their geographically closer countries Italy and Croatia. Such an accumulation of special features in certain geographical areas of a species distribution might constitute a response to specific habitat factors (see above). However, the overall tendency of a higher frequency of punicalagin derivatives containing plants in populations originating from the midMediterranean area and the minor importance of punicalagin derivatives in the continental eastern Mediterranean area were, to some degree, reflected in the greenhouse populations that were cultivated and harvested under uniform conditions. These congruent patterns would argue more for the local presence of genetic variants or for local genetic exchange. Genetic analysis run in parallel indeed detected unique DNA sequence characteristics in the Italian, Croatian and Cypriot populations (Lukas et al., unpublished). Besides the non-gradual clustering of populations related to geography, no clustering clearly related to our designation of subtaxa (subspecies or varieties) was observed. This is in accordance with [35], who analyzed flavonoid diversity of Cypriot C. creticus in more detail and described a conspicuous population cluster comparatively poor in flavonol glycosides that was in the most western part of the island (geographically close to or within the Polis Basin) and included populations of both C. creticus varieties var. tauricus and var. creticus. Based on 52 compounds, [14] could not detect significant qualitative differences in polyphenolic compound patterns of Sardinian C. creticus subsp. corsicus, subsp. eriocephalus and subsp. creticus. The subtaxon-related, intraspecific differentiation based on essential oil and NMR data [6,34] and from genetic data [6] seems not to be reflected in the polyphenolic profiles of $C$. creticus. Within the white- and whitish-pink-flowered clade, C. monspeliensis separated clearly, whereas the other three species were not separated. The evolutionary relationships that link white- and whitish-pink-flowered species were described to be rather complex and are still unresolved in many details [1,2]. Cistus monspeliensis (sect. Ledonia Dunal), C. ladanifer (sect. Ladanium (Spach.) Gren. \& Godr.), C. parviflorus (sect. Ledonella Dunal) and C. salviifolius (sect. Ledonia Dunal) have been assigned to three different generic sections but none of these sections were subsequently supported by combined DNA sequence and pollen analysis [2]. The polyphenol profiles, as recorded during this investigation, did not provide distinctive signals in the case of three of the four species included. However, it must be considered that, due to the primary aim of this investigation, a higher proportion of the total HPLC peak area of the white- or whitish-pink-flowering species was not considered. The here neglected compounds may provide further distinctive signals for a clearer species discrimination. The authors of [11] defined, beside punicalagin, hexahydroxydiphenoylglucose, (epi)catechin, (epi)gallocatechin and an (epi)catechin-(epi)gallocatechin dimer as compounds with high discriminating power.

The overall highest antioxidative activities were observed in two species of the whiteand whitish-pink-flowered clade, C. ladanifer (mean value of $303 \mathrm{mg}$ te/g dry wt) and C. salviifolius (264 mg te/g dry wt). As expected, the antioxidative capacity correlated strongly with the total phenolic content but there was solely a moderate (punicalagin derivatives), weak (quercetin glycosides) or even no (myricetin glycosides) correlation between antioxidant activity and quantified contents of main compounds and main compound families. In particular, the weak or lacking statistical correlation between antioxidative activity and myricetin or quercetin glycosides was rather surprising. Myricetin, quercetin and some of their glycosides, especially their rhamnosides, were described to be powerful antioxidants, with an antioxidant activity similar to or slightly weaker than that of vitamin $\mathrm{E}[37,46]$. However, when comparing the plots of total, myricetin, quercetin and punicalagin derivative contents of the different species with the plot visualizing their antioxidative capacity, the patterns are obviously not congruent. This was especially obvious within C. creticus. Although strikingly poorer in these compounds, the antioxidative capacity of plant material from some cultivated C. creticus populations was comparable to that of many natural populations. These findings imply that, beside the recorded punicalagin derivatives and flavonol glycosides, further components (possibly not that sensitive to certain 
growing conditions) must be significantly involved in the antioxidative capacity of Cistus plant material. Such candidate compounds would be, e.g., hexahydroxydiphenoyl-glucose, gallocatechin, gallic acid and catechin that were, besides m-3-O-rhamnoside, identified as compounds with stronger antioxidant activity in C. incanus herbal tea infusions [36].

Within the purple-flowered clade, plant material of C. crispus (206 mg te/g dry wt) and plant material of C. albidus (142 $\mathrm{mg}$ te/g dry wt) exhibited a slightly higher and a slightly lower antioxidative activity, respectively, than that of C. creticus (170 mg te/g dry wt). Compared to the white- or whitish-pink-flowered species, the antioxidative capacity of C. creticus plant material was about half of that of $C$. ladanifer, about two thirds of that of C. salviifolius or close to that of C. monspeliensis ( $175 \mathrm{mg}$ te/g dry wt) and C. parviflorus (169 mg te/g dry wt). Direct comparisons of phenolic contents and antioxidant activity with results of previous investigations of Cistus plant material were difficult as various experimental conditions and different modes of result expression were used. The total phenolic contents determined in the course of this investigation seem to be somehow higher than those published by [47] (about $55 \mathrm{mg}$ gallic acid equivalents (gae)/g dry wt in ethanolic extracts of Tunisian C. monspeliensis and C. salviifolius), seem to somehow resemble those of [32] (about $65 \mathrm{mg}$ gae/g dry wt in an aqueous extract of Syrian C. creticus, about $70 \mathrm{mg}$ gae/g dry wt in an aqueous extract of $C$. salviifolius) or seem to be somehow lower than those predicted by [48] (about $250 \mathrm{mg}$ gae/g dry wt in an ethanolic extract of a Portuguese C. ladanifer sample), [33] (up to $115 \mathrm{mg}$ gae/g dry wt in ethanolic extracts of a Bulgarian C. creticus sample), [49] (about $500 \mathrm{mg}$ gae/g dry wt in an ethanolic extract of a Tunisian C. salviifolius leaf sample) or [39] (about $408 \mathrm{mg}$ gae/g dry wt or $335 \mathrm{mg}$ gae/g dry wt in aqueous extracts of Moroccan C. salviifolius and C. monspeliensis). When comparing C. creticus to prominent aromatic plants of the Lamiaceae family that were previously studied in our lab by the same quantification method used here, the antioxidative activity of C. creticus was higher than that of Salvia officinalis [50] or Thymus vulgaris L. [51]. However, the total phenolic content of C. creticus was comparable to that of Salvia officinalis [50] and slightly higher than that of Thymus vulgaris L. [51], indicating specific compounds of higher activity in Cistus.

In recent years, Cistus plant material and Cistus preparations have been increasingly used for diverse medicinal purposes. However, up to date, no Cistus monograph is available in the European Pharmacopoeia [52] to define target species and minimum quality parameters. Despite its long traditional use in some South European countries, Cistus is subject to the "novel food regulation" (EU 2015/2283). A certain variety locally native to northern Greece, Cistus incanus L. Pandalis herba, has been registered in the category herbal infusions [53]. Apart from pharmacies, different Cistus plant materials are often offered as a "bath additive". In the face of lacking guidelines for drug quality requirements and confusion caused by the inconsistent categorization of Cistus plant material, it is doubtful that consumers can rely on a constant active substance content and a consistent quality and purity of their health remedies. To validate the polyphenolic content and composition of currently available commercial products, 15 trade samples of coarsecut Cistus plant material from different trademarks, pharmacies and health retailers were included in our analysis. Twelve of these trade samples were originally labeled as $C$. incanus and one as C. creticus, and two further Cistus herbal "teas" had no species designation on the label. The content of water-soluble compounds of the trade samples varied highly, from nearly zero to well comparable with that of plant material collected during this investigation. This striking variability in polyphenolic compound levels might reflect differences in individual sample composition, age or sampling and storage conditions of trade batches. The authors of [31] revealed that the wooden fraction of trade samples contained only small amounts of polyphenols compared to the leafy fraction. There was also high variability concerning the qualitative composition of the aqueous extracts, what was immediately visible from the characteristic peak patterns of their chromatograms. The principal component analysis then placed six of the trade samples within or close to the $C$. creticus/C. albidus cluster, whereas the other nine samples seemed to resemble 
more the typical qualitative characteristics of white-flowering species. Small white flower pieces present in some of these trade samples subsequently confirmed our results. These outcomes led to the conclusion that (currently) trade batches of Cistus plant material can differ highly in quality and are almost certainly not designated correctly or are at least admixtures of $C$. creticus and different Cistus species. These findings should be considered when postulating, assigning or comparing pharmaceutical effects based on results gained from the analysis of few Cistus trade sample preparations.

\section{Materials and Methods}

\subsection{Plant Material}

One hundred and twenty-seven populations and 1153 individual plants of C.creticus L. (72 populations, 704 individual plants), C. albidus L. (13 populations, 131 individual plants), C. x canescens Sweet (one population, one plant), C. crispus L. (two populations, 22 individual plants), C. ladanifer L. (one population of eight plants), C. monspeliensis L. (eight populations, 89 individual plants), C. parviflorus Lam. (eight populations, 48 individual plants) and C. salviifolius L. (22 populations, 150 individual plants) and 15 trade samples from different suppliers were analyzed for this investigation. The main part of the plant material was collected in the wild from native populations in Albania, Croatia, Cyprus, France, Italy, Portugal and Spain, in autumn 2016 (Albania) or the late spring of 2017 and 2018. Except for the five Albanian populations and two Portuguese populations of $C$. crispus, the bigger part of the wild plants was harvested at a comparable phenological stage (beginning to end of bloom). The second part of the analyzed plant material was from potted C. creticus (ten populations) and C. albidus (five populations) plants grown in the greenhouse (greenhouse cultivation in winter, open land cultivation from early spring to late autumn) at the University of Veterinary Medicine Vienna. Seeds for the greenhousegrown plants were obtained from the Millenium Seedbank (Royal Botanic Gardens Kew) and the Seed Bank Berlin Dahlem. The cultivated plants were in full bloom when they were harvested in their second vegetation period (early summer 2018). Geographical locations of populations can be seen in Figure 4, geographical coordinates of the native populations and seedbank accession numbers are summarized in Table S1 (Supplementary Material) and trade samples are characterized in Table 2. The included picture gallery (Supplementary Material) offers a view of selected natural populations and potted plants. All plant material of wild populations was sampled in accordance with the guidelines of the Nagoya Protocol (https://www.cbd.int/abs / text (accessed on 15 September 2016)). Species were identified by following keys of the local floras (references are provided in Table S1, Supplementary Material). Voucher specimens of wild and cultivated populations, currently kept at the herbarium of the Institute for Animal Nutrition and Functional Plant Compounds, University of Veterinary Medicine Vienna, will be submitted to the Herbarium of the Institute of Botany, University of Vienna (WU). Copyright of pictures in the graphical abstract belongs to Johannes Novak, Corinna Schmiderer, Martina Pettighofer and, in the case of $C$. crispus, Willem van Kruijsbergen (Saxifraga Foundation; http: / / www.freenatureimages.eu (accessed on 15 October 2020). 


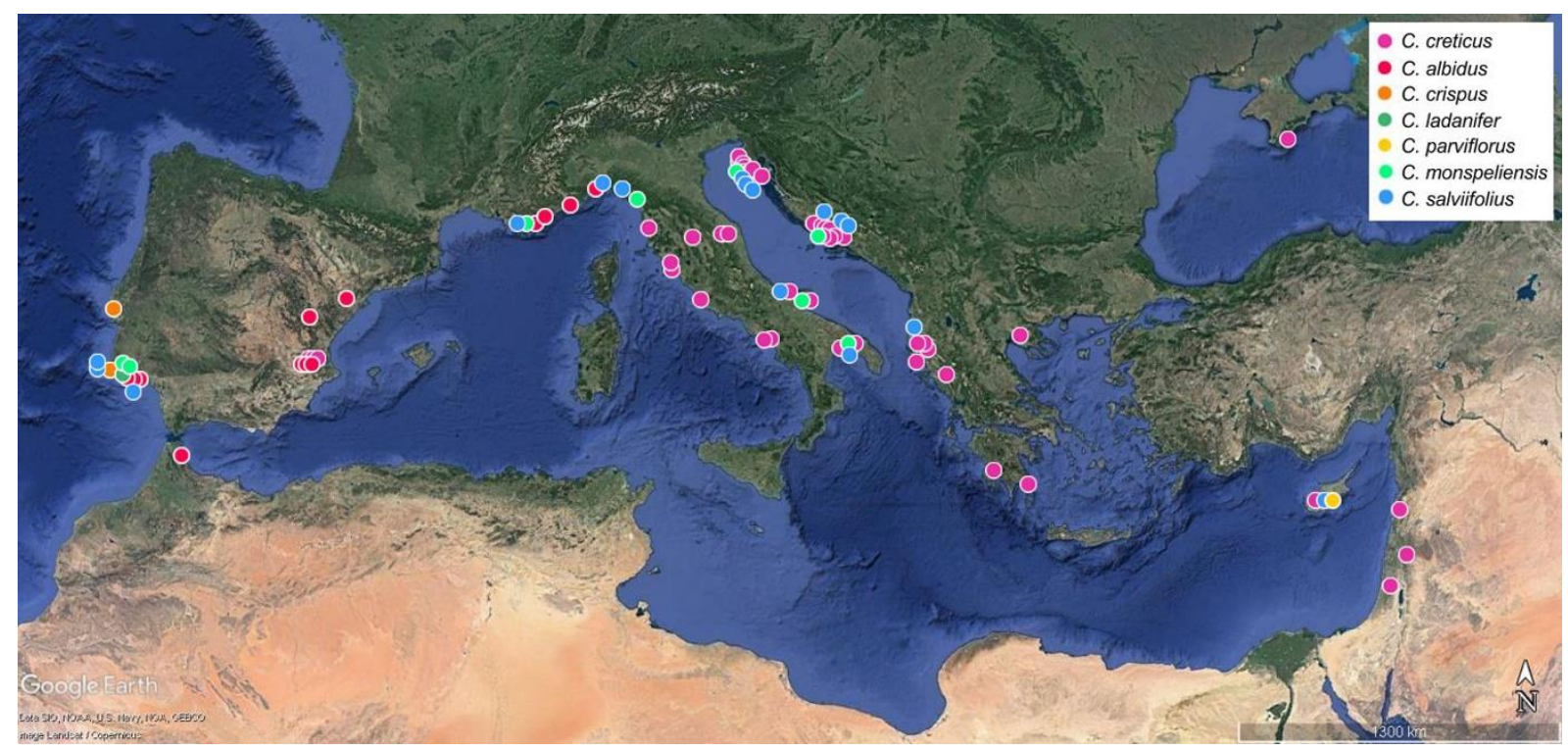

Figure 4. Geographical origin of the Cistus populations analyzed (distribution map was compiled with Google Earth (https:/ / www.google.com/earth/download/ (accessed on 12 March 2020)). The three marks in Cyprus represent the 29 populations of $C$. creticus, eight populations of $C$. parviflorus and seven populations of $C$. salviifolius sampled (mainly) in Southern Cyprus. Geographical coordinates are summarized in Table S1, Supplementary Material.

\subsection{Sampling Procedure and Handling of Plant Material}

From each individual sampled plant, one representative branch was collected from the canopy top. The plant material was either air dried (wild populations) or dried in a drying cabinet $\left(30^{\circ} \mathrm{C}\right.$, greenhouse populations). The dry plant material was then kept in cartons at room temperature. For analysis, all leaves of a branch were separated from the stems. Stems and, when present, flowers and early fruits were removed. Immediately before extraction, a representative portion of the roughly crushed leaves was ground to a fine powder by using a ball mill (Pulverisette, Fritsch, Germany).

\subsection{Extractions}

One hundred and fifty $\mathrm{mg}$ of finely grounded plant material was extracted with eight $\mathrm{ml}$ milli-Q water at $60{ }^{\circ} \mathrm{C}$, for $120 \mathrm{~min}$ in a shaking water bath (based on the protocol of [11]). The filtered extracts were aliquoted in HPLC vials (for HPLC analysis) and in $1.5 \mathrm{~mL}$ Eppendorf tubes (for analysis of DPPH and total phenolics). The aliquots were kept at $-20^{\circ} \mathrm{C}$ until analysis.

\subsection{HPLC and HPLC-MS Analysis}

HPLC analyses were performed using a Shimadzu Nexera XR chromatograph (Shimadzu, Austria) equipped with a controller (CBM-20A), a degasser (DGU-20A5R), a quaternary pump (LC-20ADXR), an autosampler (SIL-20AXR), a column oven (CTO-20AC) and a photodiode array detector (SPD-M20A). The software package LabSolutions 5.82 (Shimadzu, Austria) was used for data collection and processing. Separations were performed on a XBridge ${ }^{\mathrm{TM}}$ Shield RP18 column ( $3.5 \mu \mathrm{m}, 4,6 \times 150 \mathrm{~mm}$; Waters, Austria) equipped with a C18 guard column (ODS Octadecyl, $4 \mathrm{~mm} \times 0.3 \mathrm{~mm}$, Phenomenex, Germany). A linear gradient elution was carried out at a flow rate of $1 \mathrm{~mL} / \mathrm{min}$ and an oven temperature of $25{ }^{\circ} \mathrm{C}$ using acetonitrile (Carl Roth, Germany; solvent A) and 2\% acetic acid (Carl Roth, Germany; solvent B). The following gradient was used: min 0-38, 6-17\% A in B (linear gradient); $\min 38-53,17-20 \% \mathrm{~A}$ in $\mathrm{B}$ (linear gradient); $\min 53-58,100 \% \mathrm{~A}$ (isocratic); $\min$ $58-65,0-6 \%$ A in B (linear gradient). Injection volume was $20 \mu \mathrm{L}$. Peak detection was performed at $354 \mathrm{~nm}$, close to the UV/Vis absorption maximum of many flavonoids. 
After visual inspection of the first 30 chromatograms of Cypriot C. creticus, 13 prominent and/or recurrent peaks were defined and subsequently evaluated (Table 1 and Figure S1, Supplementary Material). Identification and verification of trans-species occurrence of these prominent compounds were conducted via literature data (references are provided in Table 1), by comparing retention times and UV spectra to those of available reference chromatography standards (punicalagin, myricetin-3-O-rhamnoside, quercetin-3-Orutinoside, quercetin-3-O-galactoside, quercetin-3-O-glucoside, quercetin-3-O-rhamnoside; all from Phytolab, Germany) and by comparative runs of selected accessions of the seven Cistus species, commercial standards and characterized green tea samples on a HPLCMS (Waters, Austria) (Table 1). The HPLC-MS was equipped with a separation module (Waters 2695), a photodiode array detector (Waters 996) and a mass spectrometer (Waters Micromass Quattro micro ${ }^{\mathrm{TM}}$ ). Various runs using alternative columns, solvents/gradients and scan modes were performed and comparatively analyzed to trace selected (mass) components and to verify the presence or composition of multiple peaks. The ESI source was operated in negative mode using the following conditions: capillary voltage $2.5 \mathrm{kV}$, cone voltage $35 \mathrm{~V}$, extractor $3 \mathrm{~V}$, RF lens $0 \mathrm{~V}$, source temperature $150{ }^{\circ} \mathrm{C}$, desolvation temperature $350{ }^{\circ} \mathrm{C}$. Nitrogen was set at $600 \mathrm{~L} / \mathrm{min}$. The unambiguous identification of smaller or minor peaks, however, was finally hindered by the combination of low signal strength and slight retention time shifts and pattern shifts when comparing HPLC and HPLC-MS chromatograms.

The quantification of punicalagin and punicalagin gallate as well as that of main myricetin and quercetin glycosides was conducted by comparison with external standards of punicalagin $\left(y=3345451 x, r^{2}=0.995 ; \mathrm{LOD}=0.189 \mu \mathrm{g} / \mu \mathrm{L}, \mathrm{LOQ}=0.631 \mu \mathrm{g} / \mu \mathrm{L}\right.$; quantification of punicalagin gallate was performed equivalent to punicalagin), myricetin3-O-rhamnoside ( $\mathrm{y}=36317204 \mathrm{x}, \mathrm{r}^{2}=0.998$; $\mathrm{LOD}=0.0199 \mu \mathrm{g} / \mu \mathrm{L}, \mathrm{LOQ}=0.066 \mu \mathrm{g} / \mu \mathrm{L}$; quantification of $\mathrm{m}-3$-O-galactoside, $\mathrm{m}$-3-O-glucoside and $\mathrm{m}$-O-xyloside was performed equivalent to $\mathrm{m}-3-\mathrm{O}$-rhamnoside) and quercetin-3-O-rhamnoside ( $\mathrm{y}=42551527 \mathrm{x}, \mathrm{r}^{2}=0.999$; $\mathrm{LOD}=0.009 \mu \mathrm{g} / \mu \mathrm{L}, \mathrm{LOQ}=0.032 \mu \mathrm{g} / \mu \mathrm{L}$; quantification of $\mathrm{q}-3-O$-rutinoside, $\mathrm{q}-3-\mathrm{O}$ galactoside, q-3-O-glucoside and q-O-xyloside or q-3-O-arabinoside was performed equivalent to q-3-O-rhamnoside). Quantification was expressed as milligram per gram dry weight (mg/g dry wt).

\subsection{Total Phenolics}

The total phenolic content was evaluated as described in [50], with small modifications. The water extracts were diluted with milli-Q water $(1: 10)$. Ten $\mu \mathrm{L}$ of the dilution was mixed with $100 \mu \mathrm{L}$ of milli-Q water and $5 \mu \mathrm{L}$ of Folin-Ciocalteu's phenol reagent (Merck, Germany) in a microplate well. The mixture was kept at room temperature for $3 \mathrm{~min}$ and then $10 \mu \mathrm{L}$ of $\mathrm{Na}_{2} \mathrm{CO}_{3}$ solution (Carl Roth, Germany; $35 \mathrm{~g}$ in $100 \mathrm{~mL}$ milli-Q water) and $125 \mu \mathrm{L}$ of milli-Q water were added. After $60 \mathrm{~min}$ of incubation in the dark, the absorbance at $750 \mathrm{~nm}$ was measured using a microplate reader (i-Mark, Bio-Rad, Austria). Caffeic acid (Sigma-Aldrich, Austria; $10 \mathrm{mg}$ in $100 \mathrm{~mL}$ milli-Q water) was used as standard. A blank was used to correct the readings. Calibration points and samples were pipetted and measured as quadruplicates. The results were expressed as milligram of caffeic acid equivalents per gram dry weight (mg cae/g dry wt).

\subsection{DPPH Radical Scavenging Activity}

The DPPH radical scavenging activity was evaluated according to [50]. The water extracts were diluted with milli-Q water (1:10). One hundred and five $\mu \mathrm{L}$ of the dilution was then mixed with $95 \mu \mathrm{L}$ methanol (Carl Roth, Germany) and $100 \mu \mathrm{L}$ of solution (2,2diphenyl-1-picrylhydrazyl, Sigma-Aldrich, Germany; $0.0038 \mathrm{~g}$ in $25 \mathrm{~mL}$ methanol). After 30 min incubation in the dark at room temperature, the absorbance of the reaction mixture was measured at $490 \mathrm{~nm}$ using a microplate reader (i-mark, Bio-Rad, Austria). Trolox (6-hydroxy-2,5,7,8-tetramethylchroman-2-carboxylic acid, Sigma-Aldrich, Austria; $0.0063 \mathrm{~g}$ in $10 \mathrm{~mL}$ pure ethanol) was used as standard. A blank was used to correct all readings. 
Calibration points and samples were pipetted and measured as quadruplicates. The results were expressed in milligram Trolox equivalents per gram dry weight (mg te/g dry wt).

\subsection{Statistics}

Statistical analyses (basic statistical parameters, correlations, Analysis of Variance (ANOVA), Tukey honestly significant difference (HSD) test, principal component analysis (PCA)) were performed and visualized by using R 3.5.2 [54] and the packages agricolae, corrplot, dplyr, factoextra, FactoMiner, ggplot2, ggpubr, ggsci, Hmisc, multcompView, PerformanceAnalytics, readxl, tidyverse and RColorBrewer.

\section{Conclusions}

Cistus creticus exhibited an impressive diversity in total content of water-soluble compounds and in contents of punicalagin derivatives and flavonol glycosides. Two chemovariants based on the presence/absence of punicalagin derivatives were identified: a more frequent one containing punicalagin derivatives and a rarer one without punicalagin derivatives. Punicalagin derivatives containing plants accumulated regionally in the western and especially the mid-Mediterranean areas and in Cyprus. In populations of the eastern Mediterranean area, punicalagin and punicalagin gallate were (almost) absent. Beside this large-scale pattern, there was no obvious correlation between polyphenolic profiles and small-scale morphological diversity supporting a classification of $C$. creticus variants to subspecies or varieties. Natural and cultivated C. creticus populations differed significantly in flavonol glycoside and punicalagin derivative contents but not in total phenolic content and antioxidative capacity. Compared to the Cistus species with the overall highest antioxidative capacity, C. ladanifer, the antioxidative capacity of $C$. creticus was approximately half. Compared to two Lamiaceae species often declared as medicinal plants with high antioxidative capacity, Salvia officinalis and Thymus vulgaris, the antioxidative capacity of $C$. creticus was higher.

The specific polyphenolic compound composition of Cistus species seems to be related to evolutionary events. Based on relative percentages of punicalagin derivatives and the main flavonol glycosides, purple-flowered and white- and whitish-pink-flowered clades could principally be well separated. Within the purple-flowering subgenus Cistus, C. crispus differentiated clearly from the strongly overlapping clusters of $C$. creticus and C. albidus. More punicalagin derivative-rich plants of C. creticus segregated towards subgenus Leucocistus and might indicate an evolutionary event differentiating western $/ \mathrm{mid}$ Mediterranean populations and eastern Mediterranean populations of C. creticus. Within subgenus Leucocistus, C. monspeliensis separated clearly, whereas the other three species did not differentiate.

Supplementary Materials: The following are available online at https:/ /www.mdpi.com/2223-774 7/10/4/615/s1, Figure S1: (a) Example chromatogram of Cypriot C. creticus recorded at $354 \mathrm{~nm}$. Red marks refer to the retention times of the 13 peaks defined after initial inspection of representative chromatograms. The peak numbers indicated in the graph correspond to the peak numbers and compounds listed in Table 1 and Table S1. (b) Direct comparison of representative example chromatograms of C. creticus (SRC580, cre), C. albidus (SRC71, alb), C. crispus (SRC135, cri), C. ladanifer (SRC59, lad), C. monspeliensis (SRC115, mon), C. parviflorus (SRC595, par) and C. salviifolius (SRC96, sal). Identification of the quantified main compounds is indicated., Figure S2: Principal Component Analysis - plot of the first two dimensions from PCA performed over all seven species and the eleven evaluated main compounds (relative area percent). Black squares indicate the position of the 15 trade samples. Figure S3: HPLC chromatograms $(354 \mathrm{~nm})$ of SRC170 and SRC204 (C. creticus, Cyrus), comparison of hydromethanolic extract (50:50; black line) and pure water extract (pink line). Table S1 (sheet 1): Geographical origin of natural and cultivated Cistus populations, collection details, number of plants analysed, population mean values and standard deviations of quantified main components, total phenolics and antioxidative activity. Table S1 (sheet 2): Species minimum values, maximum values, mean values and standard deviations of main components, total phenolics and antioxidant activity. Table S2: Statistical correlation between total phenolics (mg cae/g dry wt), antioxidant activity 
(mg te/g dry wt) and sums of punicalagin derivatives, myricetin- and quercetin-glycosides (mg/g dry wt). Picture gallery: View of selected natural C. creticus populations and potted Cistus plants.

Author Contributions: Conceptualization, B.L. and J.N.; data curation, B.L.; formal analysis, B.L., K.S. (Klaus Stolze) and J.N.; funding acquisition, B.L.; investigation, B.L., L.B., K.S. (Katharina Starzyk), K.L. and K.S. (Klaus Stolze); methodology, B.L. and J.N.; project administration, B.L.; supervision, B.L., K.S. (Klaus Stolze) and J.N.; validation, B.L., K.S. (Klaus Stolze) and J.N.; writing-original draft, B.L.; writing-review and editing, B.L., L.B., K.S. (Katharina Starzyk), K.L., K.S. (Klaus Stolze) and J.N. All authors have read and agreed to the published version of the manuscript.

Funding: Financial support for this study was provided by the Austrian Science Foundation (FWF; grant no. P 29305-B22).

Institutional Review Board Statement: Not applicable.

Informed Consent Statement: Not applicable.

Data Availability Statement: Data are contained within the article.

Acknowledgments: The authors thank the Protected Areas and Biodiversity Service of Castilla-La Mancha for authorizing the collection of samples of $C$. creticus, a protected species in Spain. The authors thank Corinna Schmiderer, Martina Pettighofer and Lina Merza for their help with plant collection in Cyprus, Italy and Croatia as well as José Gómez Navarro and Arturo Valdes for their help with collecting and herborizing plants in Spain. The authors wish to extend their gratitude to Gabriela Dekrout-Szpuszta and Bettina Bein-Lobmaier for their valuable help in the greenhouse and with plant maintenance, and to Martin Finsterböck for his technical assistance with HPLC analyses.

Conflicts of Interest: The authors declare no conflict of interest.

\section{References}

1. Guzman, B.; Vargas, P. Systematics, character evolution, and biogeography of Cistus L. (Cistaceae) based on ITS, trnL-trnF, and matK sequences. Mol. Phylogenet. Evol. 2005, 37, 644-660. [CrossRef]

2. Civeyrel, L.; Leclercq, J.; Demoly, J.-P.; Agnan, Y.; Quebre, N.; Pelissier, C.; Otto, T. Molecular systematics, character evolution, and pollen morphology of Cistus and Halimium (Cistaceae). Plant Syst. Evol. 2011, 295, 23-54. [CrossRef]

3. Von Raab-Straube, E. Cistaceae. Euro+Med Plantbase-The Information Resource for Euro-Mediterranean Plant Diversity. Available online: http:/ / ww2.bgbm.org/EuroPlusMed/ (accessed on 5 March 2021).

4. Greuter, W.; Burdet, H.M.; Long, G. Med Checklist. A Critical Inventory of Vascular Plants of the Circum-Mediterranean Countries; Conservatoire et Jardin Botanique de la Ville de Geneve: Geneve, Switzerland. Available online: http://ww2.bgbm.org/mcl/ home.asp (accessed on 5 March 2021).

5. Demetzos, C.; Anastasaki, T.; Perdetzoglou, D.A. Chemometric Interpopulation Study of the Essential Oils of Cistus creticus L. Growing in Crete (Greece). Z. Naturforsch. C 2002, 57, 89-94. [CrossRef] [PubMed]

6. Paolini, J.; Falchi, A.; Quilichini, Y.; Desjobert, J.-M.; De Cian, M.-C.; Varesi, L.; Costa, J. Morphological, chemical and genetic differentiation of two subspecies of Cistus creticus L. (C. creticus subsp. eriocephalus and C. creticus subsp. corsicus). Phytochemistry 2009, 70, 1146-1160. [CrossRef] [PubMed]

7. Brussel, D.E. Medicinal plants of Mt. Pelion, Greece. Econ. Bot. 2004, 58, 174-202. [CrossRef]

8. Lardos, A.; Prieto-Garcia, J.; Heinrich, M. Resins and Gums in Historical Iatrosophia Texts from Cyprus-A Botanical and Medico-pharmacological Approach. Front. Pharmacol. 2011, 2, 32. [CrossRef]

9. Mastino, P.M.; Marchetti, M.; Costa, J.; Usai, M. Comparison of essential oils from Cistus species growing in Sardinia. Nat. Prod. Res. 2016, 31, 1-9. [CrossRef] [PubMed]

10. Mastino, P.M.; Marchetti, M.; Costa, J.; Usai, M. Interpopulation Variability in the Essential Oil Composition of Cistus creticus subsp. eriocephalus from Sardinia. Chem. Biodivers. 2018, 15, e1800151. [CrossRef]

11. Barrajon-Catalan, E.; Fernandez-Arroyo, S.; Roldan, C.; Guillen, E.; Saura, D.; Segura-Carretero, A.; Micol, V. A Systematic Study of the Polyphenolic Composition of Aqueous Extracts Deriving from Several Cistus Genus Species: Evolutionary Relationship. Phytochem. Anal. 2011, 22, 303-312. [CrossRef]

12. Gori, A.; Ferrini, F.; Marzano, M.C.; Tattini, M.; Centritto, M.; Baratto, M.C.; Pogni, R.; Brunetti, C. Characterisation and Antioxidant Activity of Crude Extract and Polyphenolic Rich Fractions from C. incanus Leaves. Int. J. Mol. Sci. 2016, 17, 1344. [CrossRef]

13. Maggi, F.; Lucarini, D.; Papa, F.; Peron, G.; Dall'Acqua, S. Phytochemical analysis of the labdanum-poor Cistus creticus subsp. eriocephalus (Viv.) Greuter et Burdet growing in central Italy. Biochem. Syst. Ecol. 2016, 66, 50-57. [CrossRef]

14. Mastino, P.M.; Marchetti, M.; Costa, J.; Juliano, C.C. Analysis and Potential Antimicrobial Activity of Phenolic Compounds in the Extracts of Cistus creticus Subspecies from Sardinia. Nat. Prod. J. 2018, 8, 166-174. [CrossRef] 
15. Barrajon-Catalan, E.; Fernandez-Arroyo, S.; Saura, D.; Guillen, E.; Fernandez-Guiterrez, A.; Segura-Carretero, A.; Micol, V. Cistaceae aqueous extracts containing ellagitannins show antioxidant and antimicrobial capacity, and cytotoxic activity against human cancer cells. Food Chem. Toxicol. 2010, 48, 2273-2282. [CrossRef] [PubMed]

16. Petereit, F. Polyphenolische Inhaltsstoffe und Untersuchungen zur Entzündungshemmenden Aktivität der Traditionellen Arzneipflanze Cistus incanus L. (Cistaceae). Ph.D. Thesis, Westfälische Wilhelms-Universität, Münster, Germany, 1992.

17. Droebner, K.; Ehrhardt, C.; Poetter, A.; Ludwig, S.; Planz, O. CYSTUS052, a polyphenol-rich plant extract, exerts anti-influenza activity in mice. Antivir. Res. 2007, 76, 1-10. [CrossRef] [PubMed]

18. Kuchta, K.; Tung, N.H.; Ohta, T.; Uto, T.; Raekiansayh, M.; Grötzinger, K.; Rausch, H.; Shoyama, Y.; Rauwald, H.W.; Morita, K. The old pharmaceutical oleoresin labdanum of Cistus creticus L., exerts pronounced in vitro anti-dengue virus activity. J. Ethnopharm. 2020, 257, 112316. [CrossRef]

19. Rebensburg, S.; Helfer, M.; Schneider, M.; Koppensteiner, H.; Eberle, J.; Schindler, M.; Gürtler, L.; Brack-Werner, R. Potent in vitro antiviral activity of Cistus incanus extracts against HIV and Filoviruses targets viral envelope proteins. Nat. Sci. Rep. 2016, 6, 20394. [CrossRef]

20. Feng, J.; Leone, J.; Schweig, S.; Zhang, Y. Evaluation of Natural and Botanical Medicines for Activity against Growing and Non-growing Forms of B. burgdorferi. Front. Med. 2020, 7, 6. [CrossRef]

21. Rauwald, H.; Liebo, T.; Grötzinger, K.; Lehmann, J.; Kuchta, K. Labdanum and Labdanes of Cistus creticus and C. ladanifer: Anti-Borrelia activity and its phytochemical profiling. Phytomedicine 2019, 60, 152977. [CrossRef]

22. Tomas-Menor, L.; Morales-Soto, A.; Barrajon-Catalan, E.; Roldan-Segura, C.; Segura-Carretero, A.; Micol, V. Correlation between the antibacterial activity and the composition of extracts derived from various Spanish Cistus species. Food Chem. Toxicol. 2013, 55, 313-322. [CrossRef]

23. Tomas-Menor, L.; Barrajon-Catalan, E.; Segura-Carretero, A.; Marti, N.; Saura, D.; Menendez, J.A.; Joven, J.; Micol, V. The Promiscuous and Synergic Molecular Interaction of Polyphenols in Bactericidal Activity: An Opportunity to Improve the Performance of Antibiotics? Phytother. Res. 2015, 29, 466-473. [CrossRef]

24. Viapiana, A.; Konopacka, A.; Waleron, K.; Wesolowski, M. Cistus incanus L. commercial products as a good source of polyphenols in human diet. Ind. Crop. Prod. 2017, 107, 297-304. [CrossRef]

25. Hatziantoniou, S.; Dimas, K.; Georgopoulos, A.; Sotiriadou, N.; Demetzos, C. Cytotoxic and antitumor activity of liposomeincorporated sclareol against cancer cell lines and human colon cancer xenografts. Pharmacol. Res. 2006, 53, 80-87. [CrossRef]

26. Moreira, H.; Slezak, A.; Szyjka, A.; Oszmianski, J.; Gasiorowski, K. Antioxidant and Cancer Chemopreventive Activities of Cistus and Pomegranate Polyphenols. Acta Pol. Pharm. 2017, 74, 688-698. [PubMed]

27. Skoric, M.; Todorovic, S.; Gligorijevic, N.; Jankovic, R.; Zivkovic, S.; Ristic, M.; Radulovic, M. Cytotoxic activity of ethanol extracts of in vitro grown Cistus creticus subsp. creticus L. on human cancer cell lines. Ind. Crop. Prod. 2012, 38, 153-159. [CrossRef]

28. Kuchta, A.; Konopacka, A.; Waleron, K.; Viapiana, A.; Wesolowski, M.; Dabkowski, K.; Cwiklinska, A.; Mickiewicz, A.; Sledzinska, A.; Wieczorek, E.; et al. The effect of Cistus incanus herbal tea supplementation on oxidative stress markers and lipid profile in healthy adults. Cardiol. J. 2019. [CrossRef] [PubMed]

29. Attaguile, G.; Russo, A.; Campisi, A.; Savoca, F.; Acquaviva, R.; Ragusa, N.; Vanella, A. Antioxidant activity and protective effect on DNA cleavage of extracts from Cistus incanus L. and Cistus monspeliensis L. Cell Biol. Toxicol. 2000, 16, 83-90. [CrossRef]

30. Gawel-Beben, K.; Kukula-Koch, W.; Hoian, U.; Czop, M.; Strzepek-Gomolka, M.; Antowiewicz, B. Characterization of Cistus x incanus L. and Cistus ladanifer L. Extracts as Potential Multifunctional Antioxidant Ingredients for Skin Protecting Cosmetics. Antioxidants 2020, 9, 202. [CrossRef] [PubMed]

31. Wittpahl, G.; Kölling-Speer, I.; Basche, S.; Hermann, E.; Hannig, M.; Speer, K.; Hannig, C. The polyphenolic composition of Cistus incanus herbal tea and its antibacterial and anti-adherent activity against Streptococcus mutans. Planta Med. 2015, 81, 1727-1735. [CrossRef]

32. Waed, A.; Ghalia, S.; Adawia, K. Evaluation of Radical Scavenging Activity, Total Phenolics and Total Flavonoids Contents of Cistus Species in Syria. Int. J. Pharmacogn. Phytochem. Res. 2016, 8, 1071-1077.

33. Dimcheva, V.; Karsheva, M. Cistus incanus from Strandja Mountain as a Source of Bioactive Antioxidants. Plants 2018, 7, 8. [CrossRef]

34. Moosmang, S.; Sturm, S.; Novak, J.; Lukas, B.; Stuppner, H. Differentiation between Cistus L. (sub-)species (Cistaceae) using NMR metabolic fingerprinting. Planta Med. 2020, 86, 1148-1155. [CrossRef] [PubMed]

35. Lukas, B.; Bragagna, L.; Starzyk, K.; Labedz, K.; Stolze, K.; Novak, J. Polyphenol-Variabilität von zypriotischem Cistus creticus L. In Proceedings of the VIIIth Conference of Medicinal and Aromatic Plant Research, Bonn, Germany, 10-13 September 2018.

36. Barros, L.; Duenas, M.; Alves, C.T.; Silva, S.; Henriques, M.; Santos-Buelga, C.; Ferreira, I.C.F.R. Antifungal activity and detailed chemical characterization of Cistus ladanifer phenolic extracts. Ind. Crop. Prod. 2013, 41, 41-45. [CrossRef]

37. Riehle, P.; Vollmer, M.; Rohn, S. Phenolic compounds in Cistus incanus herbal infusions-Antioxidant capacity and thermal stability during the brewing process. Food Res. Int. 2013, 53, 891-899. [CrossRef]

38. Riehle, P. Phenolische Inhaltsstoffe in Cistus incanus Tee-Charakterisierung und Stabilität innerhalb der Teezubereitung. Ph.D. Thesis, University of Hamburg, Hamburg, Germany, 2014.

39. Sayah, K.; Marmouzi, I.; Naceiri Mrabti, H.; Cherrah, Y.; Abbes Faouzi, M. Antioxidant Activity and Inhibitory Potential of Cistus salviifolius (L.) and Cistus monspeliensis (L.) Aerial Parts Extracts against Key Enzymes Linked to Hyperglycemia. BioMed Res. Int. 2017, 2017, 2789482. [CrossRef] [PubMed] 
40. Baskar, V.; Venkatesh, R.; Ramalingam, S. Flavonoids (antioxidant systems) in higher plants and their response to stresses. In Antioxidants and Antioxidant Enzymes in Higher Plants; Gupta, D.K., Palma, J.M., Corpas, F.J., Eds.; Springer: Cham, Switzerland, 2018; pp. 253-268.

41. Bautista, I.; Boscaiu, M.; Lidon, A.; Llinares, J.V.; Lull, C.; Donat, M.P.; Vicente, O. Environmentally induced changes in antioxidant phenolic compounds levels in wild plants. Acta Physiol. Plant. 2016, 38, 9. [CrossRef]

42. Borges, C.V.; Minatel, I.O.; Gomez-Gomez, H.A.; Lima, G.P.P. Medicinal Plants: Influence of environmental factors on the content of secondary metabolites. In Medicinal Plants and Environmental Challenges; Ghorbanpour, M., Varma, A., Eds.; Springer: Cham, Switzerland, 2017; pp. 259-277.

43. Yang, L.; Wen, K.-S.; Ruan, X.; Zhao, Y.-X.; Wei, F.; Wang, Q. Response of Plant Secondary Metabolites to Environmental Factors. Molecules 2018, 23, 762. [CrossRef] [PubMed]

44. Gori, A.; Nascimento, L.B.; Ferrini, F.; Centritto, M.; Brunetti, C. Seasonal and Diurnal Variation in Leaf Phenolics of Three Medicinal Mediterranean Wild Species: What Is the Best Harvesting Moment to Obtain the Richest and the Most Antioxidant Extracts? Molecules 2020, 25, 956. [CrossRef]

45. Sebastini, F.; Torre, S.; Gori, A.; Brunetti, C.; Centritto, M.; Ferrini, F.; Tattini, M. Dissecting Adaptation Mechanisms to Contrasting Solar Irradiance in the Mediterranean Shrub Cistus incanus. Int. J. Mol. Sci. 2019, 20, 3599. [CrossRef] [PubMed]

46. Hopia, A.; Heinonen, M. Antioxidant activity of flavonol aglycones and their glycosides in methyl linoleate. J. Am. Oil Chem. Soc. 1999, 76, 139-144. [CrossRef]

47. Mahmoudi, H.; Aouadhi, C.; Kaddour, R.; Gruber, M.; Zargouni, H.; Zaouali, W.; Ben Hamida, N.; Ben Nasri, M.; Ouerghi, Z.; Hosni, K. Comparison of antioxidant and antimicrobial activities of two cultivated Cistus species from Tunisia. Biosci. J. Uberlandia 2016, 32, 226-237. [CrossRef]

48. Andrade, D.; Gil, C.; Breitenfeld, L.; Domingues, F.; Duarte, A.P. Bioactive extracts from Cistus ladanifer and Arbutus unedo L. Ind. Crop. Prod. 2009, 30, 165-167. [CrossRef]

49. Rebaya, A.; Belghith, S.; Cherif, J.; Trabelsi-Ayadi, M. Total Phenolic Compounds and Antioxidant Potential of Rockrose (Cistus salviifolius) Leaves and Flowers Grown in Tunisia. Int. J. Pharmacogn. Phytochem. Res. 2016, 8, 327-331.

50. Lamien-Meda, A.; Nell, M.; Lohwasser, U.; Börner, A.; Franz, C.; Novak, J. Investigation of antioxidant and rosmarinic acid variation in the sage collection of the genebank in Gatersleben. J. Agric. Food Chem. 2010, 58, 3813-3819. [CrossRef] [PubMed]

51. Chizzola, R.; Michitsch, H.; Franz, C. Antioxidative properties of Thymus vulgaris leaves: Comparison of different extracts and essential oil chemotypes. J. Agric. Food Chem. 2008, 56, 6897-6904. [CrossRef] [PubMed]

52. Council of Europe. European Pharmacopoeia, 10th ed.; Council of Europe: Strasbourg, France, 2019.

53. European Commission. Union List of Novel Foods in Accordance with Regulation (EU) 2015/2283 of the European Parliament and of the Council on Novel Foods. Available online: https:/ / eur-lex.europa.eu/legal-content/EN/TXT/PDF/?uri=CELEX: 32017R2470\&from=EN (accessed on 5 March 2021).

54. R Core Team. R: A Language and Environment for Statistical Computing; R Foundation for Statistical Computing: Vienna, Austria; Available online: https:/ / www.R-project.org (accessed on 15 September 2018). 